\title{
Physical Properties of Liquid Crystalline Solution of Once-Broken Rod Poly( $\gamma$-benzyl L-glutamate)
}

\author{
Toshinori Imai, Yoshiko Uematsu,$^{\dagger}$ Kazuyuki Hiraoka, and Munehiro Date \\ Faculty of Engineering, Tokyo Institute of Polytechnics, \\ 1583 Iiyama, Atsugi, Kanagawa 243-0298, Japan
}

(Received December 20, 2000; Accepted April 9, 2001)

\begin{abstract}
Poly $(\gamma$-benzyl L-glutamate) (PBLG) whose molecule is a straight rod in shape and a large permanent dipole moment along the rod axis and once-broken (OB)-PBLG which has two anti-parallel permanent dipoles in its molecule composed of two $\alpha$-helices jointed by a flexible chain of the bifunctional initiator (trimethylenediamine) in the manner of head to head, were synthesized and the behavior of concentrated solutions were investigated. The solution of OB-PBLG formed a cholesteric liquid crystal as well as that of straight (S)-PBLG. Temperature and concentration dependency of cholesteric texture for both polymers were similar, except for OB-PBLG having a lower molecular weight, $M_{v}<5 \times 10^{4}$. Alignment of molecules in a thin cell is different for the two polymers. As dynamics of the electro-optic response, differences for the two polymers are clearly evidently. Response time of OB-PBLG is 60 to 70 times as long as that of ordinary S-PBLG, under similar experimental conditions.
\end{abstract}

KEY WORDS Once-Broken Rod / Poly( $\gamma$-benzyl L-glutamate) / Liquid Crystalline Texture /

Electro-Optic Response / In-Plane Cell /

Poly- $\gamma$-benzyl L-glutamate (PBLG) forms a liquid crystal (LC) in a helicogenic organic solvents above a certain concentration, $C_{\mathrm{A}} \cdot{ }^{1,2}$ The theories ${ }^{3-5}$ of transition between isotropic and anisotropic phases were developed based on geometry of rod like particles, that is, axial ratio of rods, and neglecting special interactions between polymer-solvent and polymer-polymer. The cholesteric liquid crystalline texture formed by PBLG 1,2 has been shown the norm due to the optical activity of the PBLG molecule, and nematics under some particular conditions are rather exceptional. ${ }^{6-9}$

Since PBLG molecules in helical conformation have large permanent dipole moment along the helical axis, alignment of dipoles in the cholesteric texture and properties in an electric field are very interesting. The texture and properties of the liquid crystal formed by oncebroken rods, ${ }^{10-15}$ where two rods having a large permanent dipole respectively are connected in head to head manner with a hinge, the dipoles along a molecular axes are anti-parallel with each other, and may swing or rotate on the hinge in the dilute solution were studied. We prepared once-broken PBLG (OB-PBLG) introducing a hinge, as initiator, between two helical rods, and investigated the liquid crystalline texture and properties with reference to those of ordinary, single straight rod, PBLG (S-PBLG).

To make clear the dipolar interaction between molecules in the liquid crystalline phase, solutions of OB- and S-PBLG were prepared by several helicogenic solvents having different dielectric constants and differ- ences of the liquid crystal formation, texture and properties were investigated.

\section{EXPERIMENTAL}

Two PBLGs were prepared as follows. To obtain a narrow distribution of molar mass, the $N$ carboxyanhydride (NCA) of $\gamma$-benzyl L-glutamate $(\gamma$ BLG-NCA) was initially polymerized in dimethylformamide (DMF) containing $n$-butylamine for S-PBLG and trimethylenediamine (TMDA) for OB-PBLG ${ }^{10}$ as initiators respectively, fixing the mole ratio of NCA to initiator, $\mathrm{A} / \mathrm{I}$, at 50 . This solution (containing the pre-formed polymer) was added to dioxane containing the appropriate amount of NCA, to obtain the desired molecular weight of polymer. The solutions were left standing over night at room temperature and subsequently poured into a large volume of methanol. After repeating recrystallization, a suitable mass of polymer was dissolved in dioxane, 1,2-dichloroethane and 1,1,2trichloroethane respectively to prepare the sample solutions.

Molecular weights of synthesized polymers were estimated from intrinsic viscosity determined at $25^{\circ} \mathrm{C}$, in dichloroacetic acid (DCA) and DMF by equations for S-PBLG: $[\eta]=1.02 \times 10^{-4} \mathrm{M}^{0.76}$ for DCA solution and $[\eta]=5.82 \times 10^{-8} \mathrm{M}^{1.45}$ for DMF solution. DCA and DMF freshly distilled were used.

Liquid crystalline texture was observed under a desired temperature by polarizing microscope on which

${ }^{\dagger}$ To whom correspondence should be addressed (Tel and Fax: +81-462-42-9526, E-mail: uematsu@chem.t-kougei.ac.jp). 
a hot stage was mounted. The sense of cholesterics was determined by optical rotatory dispersion (ORD) and circular dichroism (CD). X-Ray diffraction was obtained with $\mathrm{Cu}-K_{\alpha}$ for the dioxane solution confined in a capillary having $1 \mathrm{~mm}$ internal diameter. The cell used for observation of director orientation by applying an electric field was constructed of two glass plates partially coated with Au thin-film as electrodes (full experimental set-up shown in ref 16). The direction of the electric field was almost parallel to the cell surface, since the ratio of cell thickness to the distance between electrodes was sufficiently small.

\section{RESULTS AND DISCUSSION}

\section{The Molecular Conformation}

Molecular weight and conformation of OB-PBLG were estimated from intrinsic viscosity of random coil conformation in DCA and helical conformation in DMF. Intrinsic viscosity was obtained from extrapolated $\eta_{\mathrm{sp}} / C$ and $\ln \eta_{\mathrm{r}} / C$ to $C=0$, where $\eta_{\mathrm{sp}}$ and $\eta_{\mathrm{r}}$ are specific and relative viscosity respectively and $\mathrm{C}$ is the concentration of polymer.

Molecular weights of four OB-PBLG (OBR) measured in DCA, $M_{v}$, and intrinsic viscosity measured in DMF solution, $[\eta]$ obs, are shown in Table I. According to theoretical calculation by Yu and Stockmayer, ${ }^{15}[\eta]$ for a once-broken rod molecule, where two equal length rods are independently jointed with a hinge, is $85 \%$ of $[\eta]$ for a straight rod having the same total length. That is to say, $\langle\cos \theta\rangle=0$, where $\theta$ represents an angle between two rods. Assuming a straight rod, intrinsic viscosity in DMF could be estimated from molecular weight, $M_{v}$, calculated from the intrinsic viscosity measured in DCA solution. The values are shown in Table II as $[\eta]_{\mathrm{cal}^{*}}{ }^{*}$. Since we could not acquire similar molecular weight of S-PBLG to that of OB-PBLG, the ratios of $[\eta]_{\text {obs }}{ }^{*}$ to $[\eta]_{\mathrm{cal}}{ }^{*}$ are shown in the last column of Table II, where asterisks mean values obtained

Table I. Characterization of polymers

\begin{tabular}{lcccc}
\hline Sample & Solvent & {$[\eta]$} & $M_{\mathrm{v}} \times 10^{-4}$ & $M_{\text {DMF }} / M_{\text {DCA }}$ \\
\hline \multirow{2}{*}{ OBR-I } & DCA & 0.357 & 4.91 & 0.845 \\
& DMF & 0.290 & 4.15 & \\
OBR-I' & DCA & 0.358 & 4.62 & 0.937 \\
& DMF & 0.308 & 4.33 & \\
OBR-II & DCA & 0.415 & 5.61 & 0.745 \\
& DMF & 0.362 & 4.85 & \\
OBR-III & DCA & 0.885 & 15.2 & 0.849 \\
& DMF & 1.505 & 12.9 & \\
\multirow{2}{*}{ SR-a } & DCA & 0.193 & 2.05 & 1.00 \\
& DMF & 0.104 & 2.05 & \\
\hline
\end{tabular}

in DMF. Provided $\langle\cos \theta\rangle=0$, the ratio is nearly be equal to 0.85 , while equal to 1 , if $\theta=180^{\circ}$, in accordance with the theoretical estimation. As shown in the table, OBPBLG scatter between 0.8 and 0.9 , while all S-PBLG is 1 which was within the experimental error. This implies that the synthesized OB-PBLG have all hooked shape. OB-PBLG are heterogeneous not only in molar mass, but also in location of the flexible portion incorporated in each molecule. Samples of OB-PBLG were contaminated with S-PBLG molecules.

\section{Formation of Lyotropic Liquid Crystalline Phase}

The concentration of phase separation between an isotropic and a liquid crystalline phases is a function of axial ratio of a particle. In order to investigate differences of axial ratio dependence of the phase behavior between S-PBLG (SR) and OB-PBLG (OBR), the polymer concentration, $V_{2}(\mathrm{v} / \mathrm{v})$ at A points $\left(C_{\mathrm{A}}\right)$, where a liquid crystalline phase begins to separate from an isotropic phase, were compared for solutions of S-PBLG and OB-PBLG. The results obtained in two solvents, at $10^{\circ} \mathrm{C}$ are listed in Table III. Due to illreproducibility with gelation and/or increase of turbidity, some data were omitted (the blank space in the Table III). Molecular weights obtained from the intrinsic viscosity measurements in DCA solutions are listed in the first column. The data in Table III are displayed graphically in Figure 1. In the figure, $V_{2}(\mathrm{v} / \mathrm{v})$ at $\mathrm{A}$ points for two polymers in two different solvents are plotted against degree of polymerization, DP, where the solid line shows the dependence of $V_{2}$ on DP, calculated from Flory's model ${ }^{3}$ assuming a rod shape having $12 \AA$ in diameter. $V_{2}(\mathrm{v} / \mathrm{v})$ at A points for OB-PBLG are always higher than those of S-PBLG, since OBPBLG molecule has large flexibility at its hinge. Differences in $V_{2}(\mathrm{v} / \mathrm{v})$ at A points among the two solvents

Table II. Characterization of once broken PBLG

\begin{tabular}{lcccc}
\hline & $M_{\mathrm{v}} \times 10^{-4}$ & {$[\eta]_{\mathrm{obs}}^{\mathrm{a}}$} & {$[\eta]_{\mathrm{cal}}^{\mathrm{a}}$} & {$[\eta]_{\mathrm{obs}}^{\mathrm{a}} /[\eta]_{\mathrm{cal}}^{\mathrm{a}}$} \\
\hline OBR-I & 4.91 & 0.290 & 0.369 & 0.79 \\
OBR-I' & 4.62 & 0.308 & 0.338 & 0.91 \\
OBR-II & 5.61 & 0.362 & 0.448 & 0.81 \\
OBR-III & 15.2 & 1.505 & 1.899 & 0.79 \\
\hline${ }^{\mathrm{a}}$ In DMF. & & & &
\end{tabular}

Table III. Critical concentration at A point

\begin{tabular}{lcccr}
\hline \multirow{2}{*}{ PBLG } & \multirow{2}{*}{$M_{\mathrm{v} /(\mathrm{DCA})}$} & \multirow{2}{*}{$\mathrm{DP}$} & \multicolumn{2}{c}{$V_{2}(\mathrm{v} / \mathrm{v})$ at $\mathrm{A}$ point at $10^{\circ} \mathrm{C}$} \\
\cline { 4 - 5 } & & & $\mathrm{In} 2-\mathrm{CE}$ & $\mathrm{In} \mathrm{EDC}$ \\
\hline OBR-I' & $4.62 \times 10^{4}$ & 211 & & 0.192 \\
SR-I & $4.51 \times 10^{4}$ & 206 & & 0.131 \\
OBR-II & $5.61 \times 10^{4}$ & 260 & 0.256 & 0.182 \\
SR-II & $6.6 \times 10^{4}$ & 260 & 0.210 & 0.130 \\
OBR-III & $15.2 \times 10^{4}$ & 694 & 0.152 & 0.118 \\
SR-III & $15.8 \times 10^{4}$ & 721 & 0.136 & \\
\hline
\end{tabular}




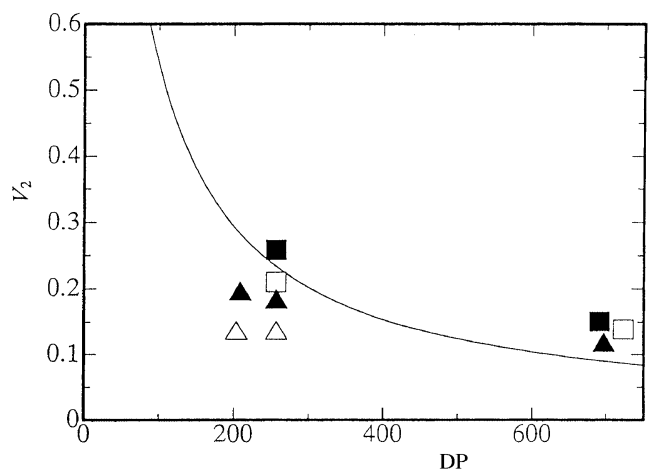

Figure 1. $V_{2}$ at A point $v s$. DP. The solid curve was obtained from PBLG concentration at A point by Flory's equation. $\triangle$ : SR/EDC, $\mathbf{\Delta}$ :OBR/EDC, $\square$ : SR/2-CE, $\mathbf{0}: \mathrm{OBR} / 2-\mathrm{CE}$.

are obvious. S-PBLG molecules associate themselves in helicogenic solvents ${ }^{17-21}$ and the type and extent of molecular association depend on the nature of solvent and concentration of polymer.

Association is roughly classified as follows ${ }^{18}: 1$ ) linear head to tail type, 2) linear head to head type, 3) side by side type with parallel orientation and 4) side by side type with anti-parallel orientation. On the basis of requirements for electrostatic free energy to be minimum, one or a combination of following two types: 1) linear head to tail type and 4) side by side type with antiparallel orientation, should be predominant mode in a helicogenic solvent. Generally, aggregation varies with solvent, which varies in dielectric constant. The relation between the type of aggregation and the dielectric constant:

1) side by side anti-parallel favor dioxane and benzene

2) head to tail favor : chloroform and 1,2-dichloroethane (EDC)

3) no aggregation : DMF, $m$-cresol

S-PBLG molecules may slightly aggregate in 2chloroetanol (2-CE) used in this measurements, having $25.8 \mathrm{D}$ of dielectric constant at $25^{\circ} \mathrm{C}$. The apparent axial ratio, $\xi$, of a particle, probably not a molecule, was calculated from $V_{2}$, polymer concentration at $\mathrm{A}$ point, according to Flory ${ }^{3}$ and plotted against DP, degree of polymerization in Figure 2. Evidently, $\xi$ in EDC is much larger than that in 2-CE and also $\xi(\mathrm{SR})>$ $\xi(\mathrm{OBR})$, where $\xi(\mathrm{SR})$ and $\xi(\mathrm{OBR})$ are apparent axial ratios of S-PBLG and OB-PBLG, respectively. Absolute $\xi$ is not important because the discrepancy between theoretical and experimental values always exists. With qualitative understanding, the ratio of $\xi$ (OBR) to $\xi$ (SR) at the similar DP was compared for each solvent. The ratio in 2-CE equals roughly 0.85 , while in EDC, nearly 0.65 . The apparent axial ratio of S-PBLG may thus increase more than that of OB-PBLG in EDC, probably owing to formation of head to tail type ag-

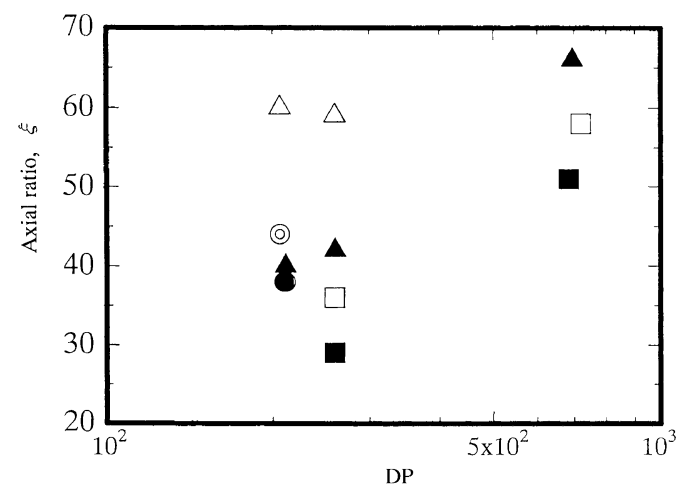

Figure 2. Apparent axial ratio of particles, calculated from $V_{2}$ at A point followed by Flory's equation. $\triangle:$ SR/EDC, $\boldsymbol{\Delta}: \mathrm{OBR} / \mathrm{EDC}, \square:$ SR/2-CE, $\mathbf{\square}: \mathrm{OBR} / 2-\mathrm{CE}, \bigcirc: \mathrm{SR} / \mathrm{EDC}+\mathrm{TFA}$, :OBR/EDC+TFA.

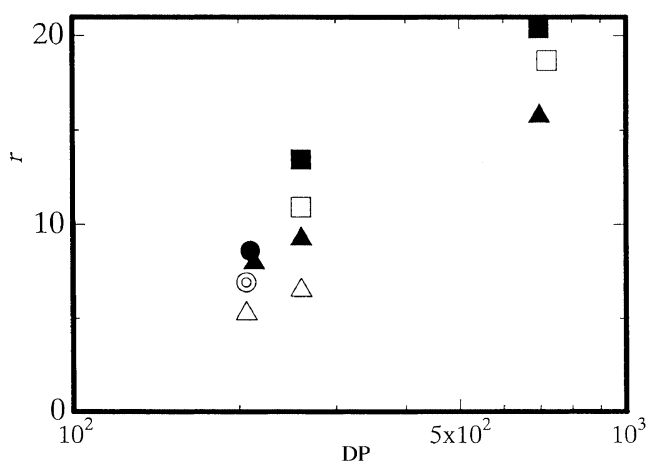

Figure 3. $r$, obtained as the ratio of $1.5 \times \mathrm{DP}$ to the apparent axial ratio. $\triangle$ : SR/EDC, $\boldsymbol{\Delta}: \mathrm{OBR} / \mathrm{EDC}, \square: \mathrm{SR} / 2-\mathrm{CE}, \mathbf{\square}: \mathrm{OBR} / 2-$ CE, (O):SR/EDC+TFA, :OBR/EDC+TFA.

gregate. As stated above, since EDC promotes head to tail aggregation, S-PBLG molecules aggregate in abundance in the manner of head to tail and then the axial ratio of particle (aggregate) formed by aggregation increase. Since OB-PBLG molecules hardly aggregate in the head to tail manner.

[If it were assumed that head-to-tail aggregation might not exist, the value of $r(r=1.5 \times \mathrm{DP} / \xi)$ would be a measure of side by side aggregation. The Figure 3 shows the variation of $r$ with DP in the different two solvents. As shown in the Figure 3, $r(\mathrm{SR})<r$ (OBR) in all solvents and the value of $r$ increases with increasing DP. If the type of aggregation is fixed, $r$ may not depend on DP. This implies that the amount of head to tail aggregation decreases with increasing molecular weight. Consequently, the fact that the A-point of S-PBLG in EDC is lower than that of OB-PBLG will provide that S-PBLG molecules form preferably head-to-tail aggregation while the fact that $r$ depend on DP even in SPBLG implies that the smaller molecule aggregates the greater in the manner of head to tail. ${ }^{19}$ For OB-PBLG, the longitudinal aggregation will be growing through side by side aggregation. The possible forms of aggregates for S-PBLG and OB-PBLG are shown in Fig- 
a

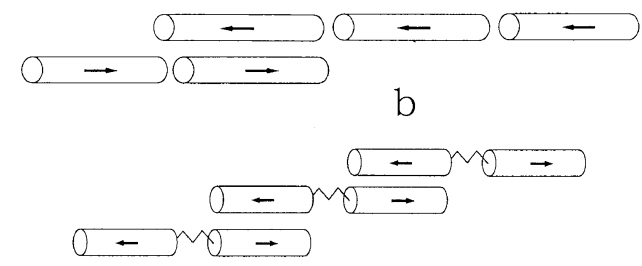

Figure 4. Mode of molecular aggregation. (a) SR, (b) OBR.

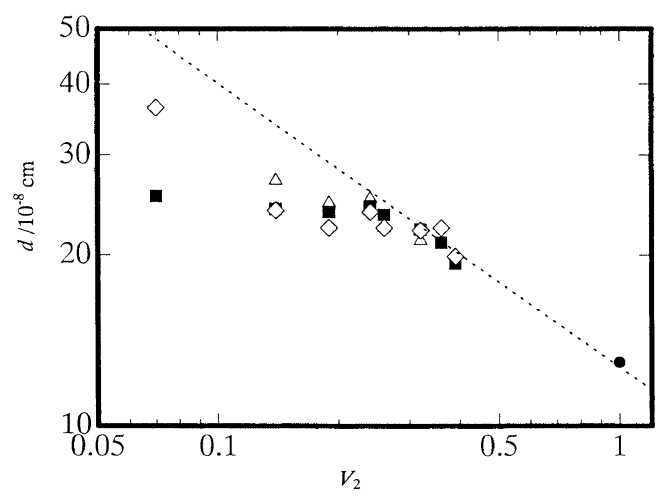

Figure 5. Intermolecular spacing $v s$. PBLG concentration in liquid crystals and in solid film. $\diamond$ : OBR-I', $\mathbf{\square}$ :SR-I, $\triangle$ :OBR-III, : OBR-I'(film).

ure 4 , schematically.]

\section{Near-Neighbor Arrangement}

According to Robinson, ${ }^{23,24}$ from X-Ray studies for dioxane solution of PBLG the molecules even in cholesteric phase, are arranged in nearly hexagonal lattice, that is, rod-like molecules of PBLG pack in something approaching a hexagonal arrangement and the cholesteric twist scarcely distorts this local ordering. Theoretical considerations of the effects of concentration of rigid rods on the distance, $d$, between the (1010) planes of the hexagonal lattice predict that $d^{2}$ is inversely proportional to the concentration of polymer, $C$, as:

$$
d^{2}=\frac{w \sqrt{3} \times 10^{23}}{2 N_{0} h C}
$$

where $w=218$, the residue molecular weight in PBLG; $N_{0}$ is Avogadro's number; $h=1.5 \mathrm{~A}$, length of the projection of a single residue along the helical axis; and $C$ is the concentration of polymer in grams per $100 \mathrm{~mL}$.

To obtain information on molecular packing in the liquid crystalline state, for both S- and OB-PBLG, the dioxane solutions were filled in a capillary and X-Ray diffraction was observed. Experimental $d v s$. polymer concentration are compared with the theory in Figure 5. The discrepancy from the theoretical curve (solid line) appears at some concentration of polymer, $C_{\mathrm{B}},{ }^{1,2}$ due to separation of the isotropic phase from the liquid crystalline phase with dilution. Since in dioxane, the preferable aggregation is side by side with anti-parallel, the discrepancy between the behaviors of both polymers may be not obvious. In the coexistence range of isotropic phase with liquid crystalline phase, packing density in the liquid crystalline phase should be preserved at a constant value. As seen in Figure 5, $C_{\mathrm{B}}$ of S-PBLG is insignificantly lower than that of OB-PBLG and at lower than $C_{\mathrm{B}}$, interchain distance, $d$, for the latter, is slightly larger than for the former. From the packing density, the hooked shape of molecule might prevent packing of molecules in the concentration range lower than $C_{\mathrm{B}}$, while unwinding of the helix part neighboring hinge may increase the flexibility of molecule and promote the parallel alignment of rods. That flexibility increases with unwinding of helix must be taken into consideration in the range of higher than it. However, since it is impossible to introduce chlorinated hydrocarbons as solvent due to their unsuitability for XRay diffraction measurement, we could draw no conclusion on differences in roles of head to tail aggregation and side by side aggregation.

\section{The Cholesteric Texture}

Whether a hinged polymer such as OB-PBLG forms or not a cholesteric texture is the question. With polarizing microscopy, we found the cholesteric texture for concentrated solutions of OB- and S-PBLG and measured the cholesteric pitch directly. The cholesteric sense was estimated by the use of optical rotation (ORD) and circular dichroism (CD) measurements, for the cholesteric solutions of both polymers at various concentrations and temperatures.

For EDC solution of OB-PBLG having $15.2 \times 10^{5}$ of molecular weight, temperature dependence of cholesteric pitch was similar to that of S-PBLG. Cholesteric pitch, $p$, increased with temperature. For the solution of OB-PBLG having $4.62 \times 10^{4}$ of molecular weight, the temperature dependence was reversed. The temperature dependence of inverse cholesteric pitch $\left(p^{-1}=2 \pi \phi / d, \phi\right.$ : the twist angle per molecule about the cholesteric axis) is shown in Figure 6, taking account of twisting sense, for EDC solutions of OBR-I and III (refer to Table I). The sense of LCICD due to phenyl residue is usually positive for the EDC solution of S-PBDG, which has $c a .5 \times 10^{4}$ of molecular weight or so, at room temperature. S-PBDG (poly- $\gamma$-benzyl Dglutamate) shows the opposite sense, i.e., negative, of LCICD to S-PBLG under the same experimental condition. OBR-III which has a large molecular weight, $c a$. $1.5 \times 10^{5}$ exhibits the same sense of LCICD as that for S-PBDG, while OBR-I which molecular weight is as small as $4.6 \times 10^{4}$, shows the opposite sense of LCICD to that for S-PBLG and then has the same sense as for 


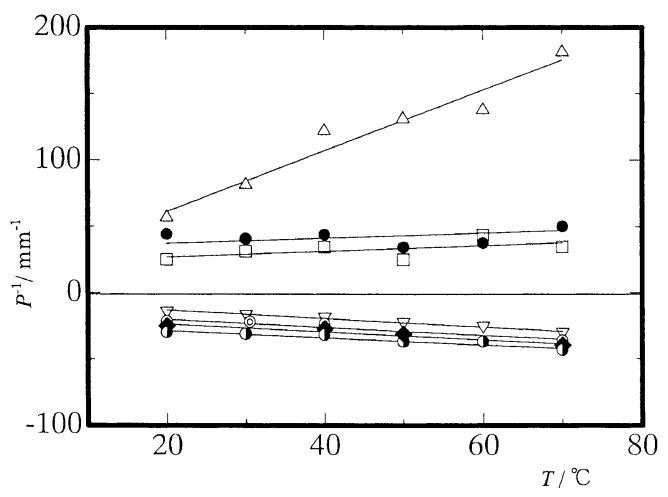

Figure 6. Dependence of cholesteric pitch on concentration of PBLG and temperature. For OBR-I/EDC, $V_{2}=0.32: \triangle, 0.38:$ 0.43: $\square$, and for OBR-III/EDC, $V_{2}=0.164: \nabla, 0.190: \bigcirc, 0.213:$ 0.282: $\bigcirc$.

(a)

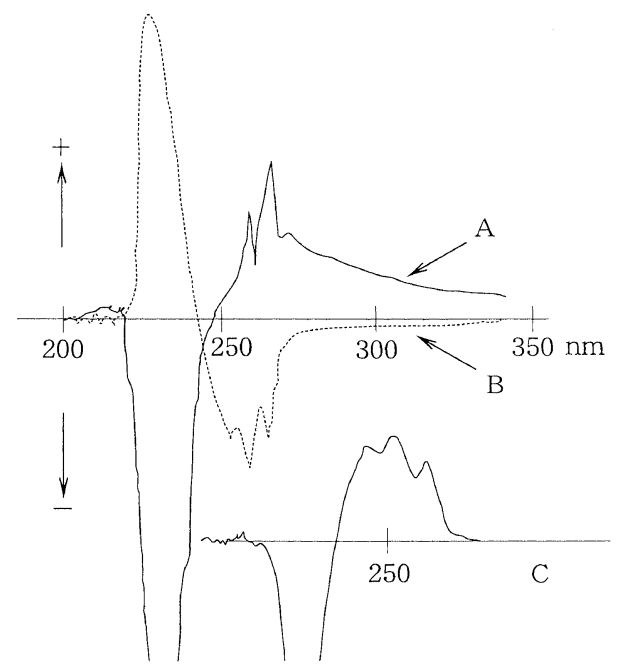

(b)

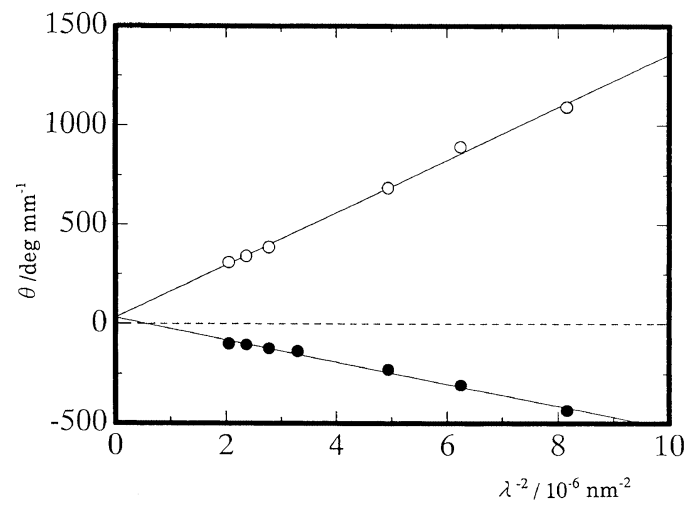

Figure 7. (a) Optical rotation as function of wavelength for OBR-I' and SR-I. $\bigcirc$ : OBR-I', $V_{2}=0.32$, $\mathrm{O}$ : SR-I, $V_{2}=0.32$; cell thickness: $0.20 \mathrm{~mm}$. (b) LCICD of PBLG-EDC and PBDGEDC. A: OBR-I' $V_{2}=0.38$; B:OBR-III, $V_{2}=0.14$, and C: PBDG $\left(M_{\mathrm{v}}: 105000\right), V_{2}=0.18$.

S-PBDG, which has the similar molecular size to OBRI, as shown in Figure 7a.

Cholesteric sense was supported by ORD measurement. In Figure 7b, the wavelength dependence of optical rotation is shown for EDC solutions of S-PBLG and OB-PBLG, both of which have about the same molec- ular weight, $4.51 \times 10^{4}$ and $4.61 \times 10^{4}$, respectively. The sense of optical rotation is negative for S-PBLG, while it is positive for OB-PBLG.

The cholesteric sense of OBR-I' might be compared to that of S-PBLG which has $1 / 2$ of molecular weight for OBR-I'. However, such low molecular weight for PBLG shows very low B point and then comparison of cholesteric sense between then is difficult.

The behavior of OBR having low molecular weight less than $5 \times 10^{4}$ is thus exceptional. As shown in Figure 6 , The temperature dependence of $p^{-1}$ for OBR-I is positive, that is, the cholesteric twisting angle increases with temperature. The concentration dependence of $p^{-1}$ for OBR-I was negative, that is, the twisting angle decreased with concentration. The concentration dependence of $p^{-1}$ for OBR-III was opposite that for OBR-I; the twisting angle for OBR-III, increased with concentration. The behavior of OBR-III is analogous to those of S-PBLG including the sense of cholesteric twist. EDC solutions of all other PBLGs except for OBR-I in the cholesteric liquid crystalline state exhibit negative optical rotation. By comparison of pitch of S-PBLG and OB-PBLG having the similar molecular weight under the same condition, pitch for S-PBLG was found always larger than for OB-PBLG. The twisting angle for S-PBLG is smaller than that for OB-PBLG.

Generally, cholesteric twisting angle per molecule for S-PBLG as a rigid rod depends on molecular weight and the smaller the molecule, the smaller the cholesteric pitch at the same concentration of polymer. This implies that OB-PBLG molecule has considerably large flexibility at the hinge and one rod in the molecule acts independently of the other in the liquid crystalline solution.

We could not analyse any further the factors determining cholesteric pitch and the sense of cholesteric twist, since the origin of cholesteric twist for this system is unknown.

The nature of solvent has effect on cholesteric liquid crystalline texture formed in the more concentrated solution of PBLG. Evidence for cholesteric pitch and sense, depending on the solvent, indicates that the effect of molecular association on liquid crystalline texture should be taken into consideration.

\section{Effects of Cell Thickness on Cholesteric Texture}

We investigated the effects of cell thickness on $C_{\mathrm{A}}$, the concentration at which a liquid crystalline phase begins to separate from an isotropic phase for EDC solution of both polymers. When the solution was put into the cell constructed with two parallel glass plates, $C_{\mathrm{A}}$ shifted to lower concentration with decreasing cell thickness in S- and OB-PBLG. The director alignment 
between the polymers differed. On decreasing cell thickness, the cholesteric texture of S-PBLG changed to homeotropic nematics, while that of OB-PBLG remained in cholesteric texture but the cholesteric axis changed from parallel to perpendicular to the cell surface. Considering the molecular alignment on the surface, there are various interactions and factors to be taken into account. Specific interactions among chain ends of polymer and the cell surface, and electrostatic interaction between polymer-polymer and/or polymersolvent molecules may determine molecular alignment and the excluded volume effect or the confinement effect should be considered.

The anchoring effect of chain end, particularly amino-end (N-end), on glass surface is a factor of homeotropic alignment. In fact, when the glass surface was covered with the thin layer of non-polar polymer, the homeotropic alignment became unstable. If the main factor of homeotropic alignment is the anchoring of chain end on a glass surface, OB-PBLG molecule having two N-ends in a molecule, should exhibit stronger anchoring than S-PBLG. But the experimental results showed no effect of anchoring on the molecular alignment on the cell surface.

As shown in previous section, cholesteric twisting power is larger in OB-PBLG than in S-PBLG under the same experimental conditions. To transform the cholesteric to the homeotropic nematic, greater energy is needed for OB-PBLG compared to S-PBLG. Cholesteric texture remains in OB-PBLG under certain experimental conditions, while, in S-PBLG, the cholesteric transforms to the homeotropic nematic under the same conditions.

For S-PBLG, taking the planar orientation of molecules, it should be difficult to fill the thin space on the cell surface with rod-like molecules without defects. If molecules are homeotropically aligned, it is possible to fill the limited thin space with molecules in the manner of hexagonal packing where the molecular axes align themselves perpendicular to the glass surface and satisfy the condition of minimum electrostatic free energy.

The alignment of polymer molecules in the vicinity of the cell surface is proposed in Figure 8. Packing density in the homeotropic alignment is larger than that in planar alignment. For OB-PBLG, if molecules align homeotropically, the requirement for electrostatic free energy to be minimum, could not be satisfied and planar alignment of molecules must be inevitable. If homeotropic alignment is taken, packing density decreases, as shown in Figure 8b.

In a comparably thin cell, alignment of molecules closed to the cell surface will be conveyed to the bulk of a
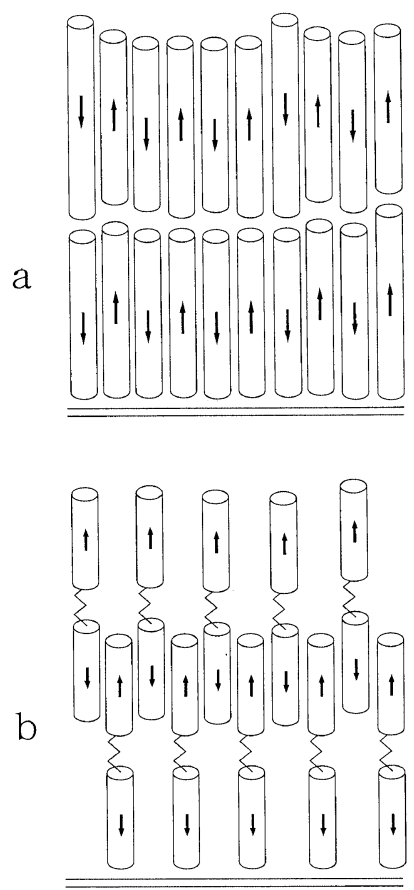

Figure 8. Molecular alignment on a glass plate. (a) SR, (b) OBR.

the cell. Thus, not only anchoring but also confinement should be taken into account for molecular alignment in a thin cell.

\section{Electric Field Effects}

We investigated molecular orientation in an electric field for S-PBLG liquid crystalline solution by in-plane type of cell and found that transient band texture as regular stripes perpendicular to the field direction appeared with inversion of the field direction. ${ }^{16}$ In adjacent bands the director rotated mutually clockwise and counterclockwise. It is interesting that the electrooptic behavior of OB-PBLG molecule, which has the two dipoles, connected antiparallel with flexible short chain, whether OB-PBLG molecule in the liquid crystalline state made response to an electric stimulation or not. If it does, the behavior must be quite different from that of S-PBLG. However, the band texture was observed in OB-PBLG solution as well as in S-PBLG, but the domain boundary in the former is not straight but zigzag and the band width, $L$, of the former is larger than that of the latter. The rate of director rotation under the same experimental condition is quite different for the two polymers. Variation in light intensity transmitted through the cell between crossed polarizers was monitored by a photo multiplier attached to a microscope. The upper polarizer was placed perpendicular to the field direction. There appear two peaks of optical transmittance in a perfect turn of director. The period of time to emergence of the first peak of transmittance after turning of the field direction, $t$, is supposed to be a 
Table IV. Band width $/ \mu \mathrm{m}$

\begin{tabular}{lcccccc}
\hline Field strength $/ V \mathrm{~cm}^{-1}$ & 300 & 250 & 200 & 150 & 100 & 50 \\
\hline SR & 12 & 13 & 16 & 22 & 23 & 30 \\
OBR & $28 \pm 2$ & $35 \pm 6$ & $39 \pm 5$ & $45 \pm 6$ & $46 \pm 2$ & $54 \pm 2$ \\
OBRSR & 2.3 & 2.6 & 2.4 & 2.0 & 2.0 & 1.8 \\
\hline
\end{tabular}

Table V. Tine 1 st peak appears after field inversion, $t / \mathrm{s}$.

\begin{tabular}{lcccccc}
\hline Field strength $/ V \mathrm{~cm}^{-1}$ & 300 & 250 & 200 & 150 & 100 & 50 \\
\hline SR & 7.2 & 8.8 & 11.8 & 22.8 & 40.2 & 76.2 \\
OBR & 530.1 & 636.0 & 885.6 & 1680 & 2325 & 4560 \\
OBRSR & 73 & 72 & 75 & 74 & 57 & 59 \\
\hline
\end{tabular}

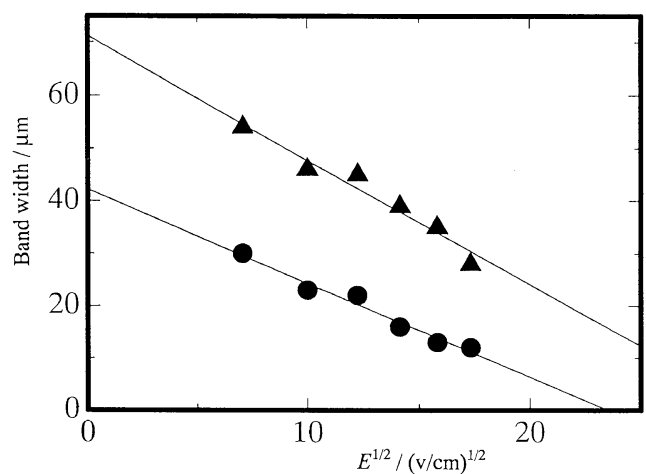

Figure 9. Band width as function of applied field strength for OBR-II and SR-II; solvent: 1,1,2-trichloroethane, concentration: $V_{2}=0.32$. $\bigcirc$ : SR-II, $\boldsymbol{\Delta}:$ OBR-II.

measure of relaxation time, that is, the time is inversely proportional to rate of director rotation in relaxation.

$L$ was compared for two polymers, Table IV. Molecular weights of polymers used were 54000 for S-PBLG and 56000 for OB-PBLG. The polymers were dissolved in 1,1,2-trichloroethane and concentration was adjusted at $V_{2}=0.32 \mathrm{v} / \mathrm{v}$ for both polymers. Observations were carried out in the cell having $0.1 \mathrm{~mm}$ of thickness and at $25^{\circ} \mathrm{C}$. As it is seen in Figure 9, band width depends on the applied DC field, $E\left(\mathrm{~V} \mathrm{~cm}^{-1}\right)$ and decreases linearly with $E^{1 / 2}$ for both polymers. $L$ for OB-PBLG are considerably larger than for S-PBLG at the same voltage. The solid line in the figure is drawn by the least squares method. Extrapolated $L$ to $E=0, L_{0}$, should be a measure of latent domain size without field.

The period, $t$, is undoubtedly a function of $E$. Observed $t$ 's are listed in Table $\mathrm{V}$. We obtained the relation between $L$ and $t$, as $t^{(1 / 4)} \sim\left(L_{0}-L\right) / L_{0}$, as shown in Figure 10. Extrapolated $t$ to $L=L_{0}$ are the order of $2 \times 10^{2} \mathrm{~s}$ and $2 \times 10^{4}$ s for S-PBLG and OB-PBLG, respectively.

Previous results for S-PBLG may lead to the following mechanisms of band formation and director rotation in the bands. The band texture is formed in relaxation after inversion of electric field direction. Before the inversion, not only dipoles but also molecular directors align parallel to the field direction. Energy, other than electrostatic energy, due to parallel and untwisted align-

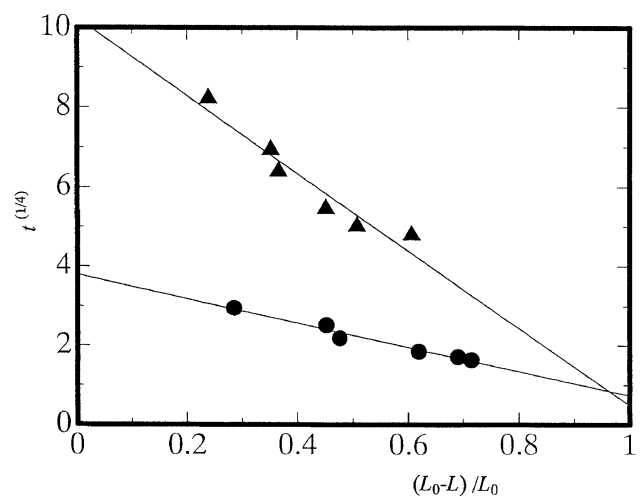

Figure 10. Period, $t, v s$. band width. $t$ is time needed to make the director rotate from 0 to $45^{\circ}$ after inversion of the electric field direction. $L_{0}$ is bandwidth extrapolated to $E=0$.

ment of molecular axes, because of the chiral character specific to polypeptide molecules, is additionally accumulated. After the inversion, the energy should be dissipated during band formation.

The rotational rate of the director must depend on the correlation radius of dipole-dipole interaction, electric susceptibility and viscoelastic resistance of domain boundary. Large differences in rotational rates of the polymers explain the discrepancy in director orientation and the fact that even LC solution of OB-PBLG which has a pair of antiparallel dipoles in a molecule exhibits similar behavior in an electric field suggests that complete alignment of dipoles in the same direction needs not to form the band. The ratios of rotational velocity of OB-PBLG to S-PBLG are large, in the order of $60-100$.

\section{CONCLUSION}

The once broken PBLG was synthesized using TMDA as initiator and two rods in an OB-PBLG molecule freely and independently rotate about each other at the joint part in the dilute solution. The axial ratio of OB-PBLG is smaller than that of S-PBLG having the similar molecular weight.

Phase separation of the liquid crystalline phase from the isotropic solution appeared at the more concen- 
trated solution for the former than the latter in the solvents having rather high dielectric permittivity, and thus differences in the two polymers were insignificant in the solvent of lower dielectric permittivity. OB-PBLG molecules may thus assemble through antiparallel side by side aggregation, to make particles having a certain axial ratio.

From X-Ray diffraction measurement for dioxane solutions of the polymers, concentration dependence of intermolecular distance was similar. We could estimate two rods of an OB-PBLG molecule aligned nearly antiparallel in liquid crystalline phase. Cholesteric pitch and twisting sense of S-PBLG depended on temperature and concentration in similar manner as OB-PBLG, except for OB-PBLG having lower molecular weight than $c a$. $5 \times 10^{4}$, although the quantitative value of twisting, $(1 / P)$, of OB-PBLG is larger than that of SPBLG under the same experimental conditions. Molecular weight is larger and twisting angle is smaller, due to excluded volume effect. The exceptional behavior of OB-PBLG having low molecular weight might be caused by increasing flexibility on the hinge. Inter-rod interactions among neighbor rods would suppress the flexibility on the hinge between twin rods. Balance between flexibility on the hinge and inter-rod interaction should be lost with decreasing rod length. Molecular length would correspond to that of a rod rather than molecule and two rods in a molecule might behave independently to some extent in bulk liquid crystalline solution, even though in liquid crystalline phase, inter-rod interactions hindered the rotation of rods on the hinge.

Molecular orientation in very thin cell constructed with two glass plates was different for two PBLGs. Generally, for S-PBLG in EDC, the homeotropic texture appears in a considerably thin cell. The homeotropic texture is taken easier in the thinner cell and for lower molecular weight of molecule. This is considered due to interactions of the N-terminal of the chain with the glass surface and then OB-PBLG should exhibit homeotropic texture more easily compared to $\mathrm{S}$ PBLG. However the experimental results disproved this expectation. We have to take into account other effects on orientation of molecules than the intrinsic anchoring to the glass surface.

In reorientation of director in IPS (in-plane switching) cell, the band texture was generally observed for SPBLG liquid crystalline solution. Even though in OBPBLG liquid crystalline solution band texture similar to that of S-PBLG appeared, band width and reorientation rate of director were quite different to each other.
The rate of reorientation for S-PBLG was several ten times that for OB-PBLG. If twin rods in an OB-PBLG molecule are folded and aligned in parallel with each other, the rate of reorientation for OB-PBLG should be as great as S-PBLG. Thus we conclude twin rods in an OB-PBLG molecule are aligned antiparallel to each other in the liquid crystalline phase, due to intermolecular interactions and excluded volume effect and the OBPBLG molecule is extended and not folded in the liquid crystalline phase.

\section{REFERENCES}

1. C. Robinson, Trans. Faraday Soc., 52, 571 (1956).

2. C. Robinson, J. C. Ward, and R. B. Beevers, Discuss. Faraday Soc., 25, 29 (1958).

3. P. J. Flory, Proc. R. Soc. London, Ser., A239, 73 (1956); P. J. Flory, Adv. Polym. Sci., 59, 1 (1984).

4. L. Onsager, Ann. N. Y. Acad. Sci., 51, 627 (1949).

5. A. Ishihara, J. Chem. Phys., 19, 1142 (1951).

6. S. Sobajima, J. Phys. Soc. Jpn., 23,1070 (1967).

7. E. T. Samulski and A. V. Tobolsky, Macromolecules, 1, 555 (1968).

8. C. Robinson, Tetrahedron, 13, 219 (1961).

9. R. W. Duke, D. B. Dupre, W. A. Hines, and E. T. Samulsky, J. Am. Chem. Soc., 98, 3094 (1976).

10. A. Teramoto, T. Yamashita, and H. Fujita, J. Chem. Phys., 46, 1919 (1967).

11. L. Reibel, G. Spach, and C. Dufour, Biopolymers, 12, 2391 (1973).

12. H. Kihara, K. Tanno, and A. Wada, Polym. J., 5, 324 (1973).

13. K. Nakagawa, N. Nishioka, A. Teramoto, and H. Fujita, Polym. J., 4, 332 (1973).

14. T. Matsumoto, N. Nishioka, A. Teramoto, and H. Fujita, Macromolecules, 7, 824 (1974).

15. H. Yu and W. H, Stockmayer, J. Chem. Phys., 47, 1369 (1967).

16. K. Monzen, K. Hiraoka, Y. Uematsu, and M. Date, Polym. J., 30, 499 (1998).

17. J. C. Power, Jr. and W. L. Petucolas, Biopolymers, 9, 195 (1970).

18. A. K. Gupta, C. Dufour, and Marchal, Biopolymers, 13, 1293 (1974).

19. A. Gupta, Biopolymers, 15, 1543 (1976).

20. K. Tsuji and H. Watanabe, J. Colloid Interface Sci., 62, 101 (1977).

21. H. Kihara, Polym. J., 9, 443 (1977).

22. H. Watanabe, Y. Fukuda, and T. Nakano, J. Colloid Interface Sci., 108, 347 (1985).

23. C. Robinson and J. C. Ward, Nature, 180, 1183 (1957).

24. C. Robinson, J. C. Ward, and R. B. Beevers, Discuss. Faraday Soc., 25, 29 (1958). 